Article

\title{
A System Model for Personalized Medication Management (MyMediMan)—The Consumers' Point of View
}

\author{
Elena Vlahu-Gjorgievska ${ }^{1, *(D)}$, Khin T. Win ${ }^{1}$ (D) and Willy Susilo ${ }^{2}$ (D) \\ 1 Centre for Persuasive Technology and Society, School of Computing and Information Technology, \\ University of Wollongong, Northfields Ave Wollongong, NSW 2522, Australia; win@uow.edu.au \\ 2 Institute of Cybersecurity and Cryptology, School of Computing and Information Technology, \\ University of Wollongong, Northfields Ave Wollongong, NSW 2522, Australia; wsusilo@uow.edu.au \\ * Correspondence: elenavg@uow.edu.au; Tel.: +61-2-4221-4606
}

Received: 22 February 2018; Accepted: 22 March 2018; Published: 24 March 2018

\begin{abstract}
In this paper, we propose a design for a personalized medication management system model MyMediMan that provides medication information for different stakeholders. The focus of the paper is on the system's features and personalized information provided for the consumers as primary users of the proposed solution. The presented design introduces the consumers to different aspects of the medications they take and their overall health condition. The personalized information should increase the consumers' awareness about the positive benefits of taking the medications as well as the consequences that particular medication can have on their health condition. By obtaining this information, the consumers will be aware of various medications' characteristics and different ways to improve their health, and thus be more actively involved in their healthcare.
\end{abstract}

Keywords: information system; medication management; personalization; persuasive technology

\section{Introduction}

Today, we are seeing increased interest towards the adoption and application of information technology (IT). One of the fields in which the introduction of IT is rapidly developing is healthcare. Providing health information online and online health education could supplement face-to-face health education to patients [1,2].

Medication management has been identified as an important factor in chronic disease management. Consumers need to be well-informed regarding their medication and to understand their disease management [3]. Not knowing the importance of taking regular medication and forgetting to take the medication as prescribed leads to non-adherence to medication. Non-adherence is recognized as one of the major problems in health care [4]. Therefore, providing medication information and reminding consumers regarding their medication through information technology will assist patients suffering from chronic diseases with their medication management [3].

With computers becoming increasingly ubiquitous and embedded, persuasive technology gain access to areas where human persuaders would not be welcomed or are physically unable to go [5]. The intention of the persuasive technology is to design the technology for the purpose of guiding the user towards an attitude or behaviour change [6]. Persuasive technologies should be adaptive and personalized, so the successful development of adaptive persuasive systems requires a combination of design, social science, and technology [7]. Personalization or computer tailoring refers to the adaptation of the output of a persuasive technology to the individual [8]. Therefore, to create personalized persuasive technology, it is necessary to be able to estimate the susceptibility of a person to different persuasive strategies [9]. 
The focus of this paper is on providing medications' information for consumers and the way that information can be personalized. Although there are different definitions for personalization $[5,6,10,11]$, in this study we consider personalization as an assemblage of customization and tailoring. Tailoring refers to the presentation of the information to the user, and customization as the way the user interacts with the system. In addition, the personalization of the system depends on the data and information gathered for and from the user.

All identified features for providing effective and personalized information are incorporated into a new, more dynamic design for a medication management system MyMediMan. In the presented model, we use the persuasive technology in order to enhance the education of patients on healthcare and medicines, extend the expert knowledge about medications, and inform users about the latest developments in the pharmaceutical industry. The information presented to the consumer are personalized according to his/her medications, health condition and age. The main goal of the MyMediMan is to increase health literacy by introducing a different aspect of medications and thus to support consumer self-efficiency.

\section{Background}

Osborn et al. [12] noted that information is the prerequisite for the health behaviour change. Therefore, a relevant knowledge related to medication is needed for medication adherence by consumers [12]. Users need to know the medication information such as its role in the treatment, side effect and benefits. Hence, providing medication information is first and foremost important for the medication management applications.

Blom [10] defines personalization as a process that changes the functionality, interface, information content, or distinctiveness of a system in order to increase its personal relevance to an individual. This process includes the system and the user, and both sides can initialize the personalization.

In the framework for Persuasive Systems Design (PSD) [11], "tailoring" and "personalization" are identified as persuasive features of primary task support category. In this context, tailoring provides tailored information for a group of users and personalization offers personalized content and services for individual users. According to Dijkstra [8], personalization or computer-tailoring acknowledges differences between individuals and refers to the adaptation of the output of a persuasive technology to the individual.

Kaptein et al. [7] present two distinct methods, explicit and implicit, for personalization of the systems and applications. According to them, questionnaires, such as meta-judgmental measures, in which the users express their own traits, can be used for explicit personalization. On the other hand, implicit personalization can be based on behavioural responses, so the system can adapt to the users' personal needs based on the user's interactions with the system.

A type of explicit personalization is covered in [13]. Lee et al. [13] propose a reflective approach to personalization that helps people realize what matters to them and create personalized services that work best for them. The aim of this approach is to influence the ways of designing personalized environments, thus the users are active participants that have the power to create their own personalized solutions.

Tara [14] consider information tailoring as equivalent to "infomediation" which refers to any mediation activities that reshape an original information package for maximum usability and influence on the destined users. Therefore, the tailored information should attract users' greater attention, greater comprehension, greater likelihood of discussion of content with others, a greater change in cognitive behavioural mediating constructs addressed by the content, and a greater likelihood of behavioural change [15].

Similarly, message tailoring is the process of individualizing communication content based on user's characteristics such as name, gender, race/ethnicity and information related to his/her behaviour [16]. Providing health communication messages tailored according to the individual characteristics could increase the effectiveness of patient education [17]. Tailored information can be 
provided to patients as feedback and reminders [18], "tailored recommendations" [19], or tailored interactive text encouragements [8].

\section{Related Work}

There are many of systems and applications for medication management.

Granger et al. [20] developed "Digital Drag and Drop Pillbox" (D-3 Pillbox). This solution uses skill-based learning to advance medication education not only by addressing what to take but also eliciting the patients' input and participation in why, how, and when to take a medication. According to this research, using an e-tablet and the patient's own medication list is a feasible solution for patients with chronic illness in teaching medication self-management.

The main features of "Medication Assistant" [21] are the alerts for medication intake, the visualization of information of each medication and the advice service that allows users to obtain information about what to do in case of forgetting to take a medication. In order to facilitate the access by older adults, the application allows interaction by speech and touch. The aim of "Medication Assistant" is to lower the non-adherence to medication, by helping the people with medication management.

The goal of the study of Martin-Hammond et al. in [22] is to understand and explore technology features that may be beneficial for older adults. For that purpose, the authors design an interface for over the counter (OTC) medication decision-making tool. The results of this study showed that older adults found features of the prototype supportive for assisting with OTC decision-making. However, there is still a need for a more flexible design with different information, personalization and navigation preferences.

To better understand the current persuasive systems design features of available medication management information systems, Win et al. [23] conducted a literature review. According to their results, the principle of personalization is presented only in three out of thirteen reviewed articles. The personalized features included information regarding medication taking records, adherence performance and reminder messages.

Besides the solutions presented in [20-22], there are several papers that propose the integration of medication management systems with electronic health record and prescription systems [24,25]. However, all these systems mostly focus on medication adherence (medication regime) with features like reminders and alarms. Some of them introduce medication information, but usually, that information is limited to the users' prescribed medications. The missing strand in all of these is the personalization of the medications' information and additional readings.

Unlike the previously reviewed applications/systems, the solution presented in this paper (MyMediMan) offers information about different medication, health and education tips, as well as information regarding the ongoing trials and research in pharmaceutical industry. Furthermore, the information presented to the user is personalized based on the consumer's age and diagnosis.

\section{Designing Personalized System Model for Medication Management}

The presented system model MyMediMan uses information technologies in order to gather data and provide personalized information needed by the consumers. In the context of the proposed design, personalization is used for tailoring information and educational contents presented to the consumers. Thus, MyMediMan encourages the users to be informed, effectively manage their medicines and actively participate in their own healthcare.

\subsection{Interview Results}

The personalization of the system depends on the quantity of data and information gathered for and from the consumers. Even though the systems' policies ensure the maximum protection of private data, the users usually do not feel confident to store personal data in the information systems. 
In order to explore the user's opinion regarding the need for personalization of the medication management systems and the personal information the consumers are willing to store into the system, we conducted interviews with 41 participants (Table 1).

Table 1. Interview participants' opinion regarding the features and personalized information needed in MyMediMan.

\begin{tabular}{cll}
\hline Feature & & \multicolumn{1}{c}{ Characteristics } \\
\hline & - & Name \\
User profile & - & Surname \\
& - & Age \\
& - & Diagnosis \\
Customization of the user interface & - & Previous searches \\
\hline \multirow{2}{*}{ Multimedia contents } & - & Multilingual platform \\
& - & Pictures \\
& - & Graphs \\
Advanced search (by) & - & Video \\
& - & Diagnosis \\
& - & Disease symptoms \\
Social media support & - & Keywords \\
& - & Forum \\
& - & Private chat \\
& - & Link to Facebook or Twitter \\
\hline \multirow{2}{*}{ Credibility } & - & Safety certificate \\
& - & Statement of confidentiality \\
& - & Presented authorisation of the information source \\
\hline
\end{tabular}

The interviews were conducted with 20 male and 21 female participants. The participants were from different backgrounds. Twenty-four participants had solid IT knowledge, and 17 participants had a health education background. The majority of participants, 39 out of 41 , were within the age group of 21-30. Only 2 out of 41 participants are within the age group of 31-40. The interviews were focused on the features that the medication management system should include in order to better satisfy the users' needs (Table 1).

Most of the participants $(72.7 \%)$ pointed out the importance of personalization. According to their opinions, medication management system should include user profiles created with user's registration. User profile should store personal data like age and diagnosis. Also, the system should allow users' previous searches to be stored. Based on the personal information and previous searches stored in the user's profile, customization of the system's interface and resources should be done. In total, $27 \%$ of the participants proposed a multilingual system support or text-to-speech option, as additional personalization of the system.

Almost the same percentage $(72 \%)$ of the participants stressed that the system should have a good interface that allows better Human-Computer Interaction (HCI). This interaction should provide diverse search options. One of the features of the systems should be searching for different medicines and educational materials throughout an advanced search by diagnosis, disease symptoms or keywords. Also, the medications' information should include multimedia contents (pictures, graphs, video, etc.). According to the participants' opinion, visual elements make the system more attractive and acceptable for the users.

Over $63 \%$ of the participants emphasized the need for information and experience exchange between users in the form of forums, blogs and social media (Facebook, Twitter, etc.). In this context, the participants focused on the possibility the users to contact with and raise questions to the professionals-medical doctors or pharmacists.

Some of the participants $(27 \%)$ highlighted the importance of privacy and underlined that all privacy issues must be covered with policies. They stressed some characteristics that will strengthen the users' trust in the system. According to the interviewed participants, the system must have a safety 
certificate; the system should evidently display the statement of confidentiality; and the system needs to present the (authorization of) information source for the presented content.

\subsection{MyMediMan Overview}

In the proposed Personalized System Model for Medication Management MyMediMan (Figure 1), three stakeholders are distinguished: consumers, pharmacists, and pharmaceutical industries. Furthermore, the information is classified into four categories: Medication Info, Health Insights, Educational Tips, and News. The Medication Info contains general information about medications, their usage and dosage, as well as information about medication's contraindications, precautions and side effects. Additional information about conditions when the medication cannot be used, substitution and refill for the given medication are also part of the Medication Info. Health Insights and Educational Tips contain multimedia contents (newsletters, presentations, video, etc.) related to health. Health Insights consists of different contents about food supplements, diets, and physical activities that are important for people with a specific health condition. Educational Tips includes educational contents related to particular health condition. The News consists of information about the latest ongoing trials with new medications and research in the field of the pharmaceutical industry.

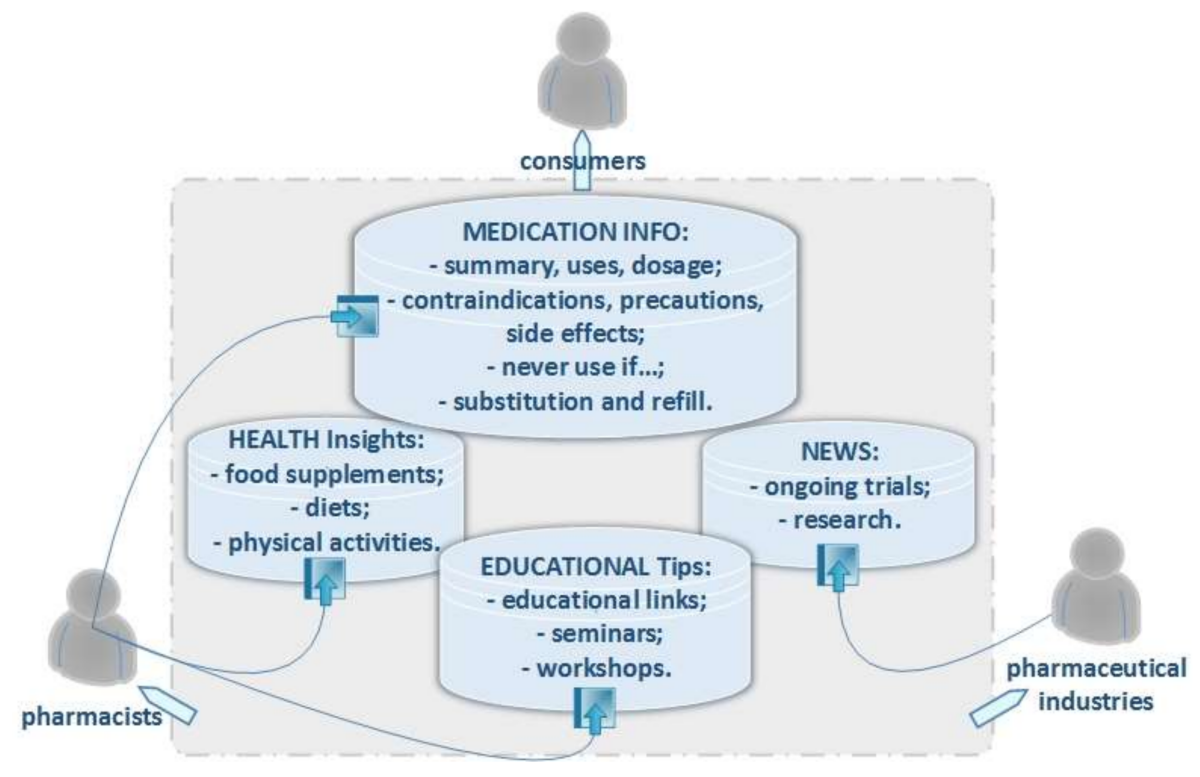

Figure 1. Stakeholders and Information categories in MyMediMan.

All three stakeholders, regarding their needs, can use the system in different ways. The consumers can search and obtain information related to the medications they are using. They can also gain additional knowledge about different medications and ways to improve their health condition. For additional information regarding their point of interest, the consumers can use the educational tips or news provided by the system.

The pharmacists are a very important loop in the system. They provide expert knowledge about medication, health, and educational tips. Also, they can use the system to gain information about the latest development in the pharmaceutical industry.

The pharmaceutical industries provide information about their latest achievements: ongoing trials and research. By sharing the information and the latest news from the pharmaceutical area with the community, the pharmacists and the pharmaceutical industry can easily access clients and get information about their concerns and needs. On the other hand, having access to the latest information regarding the pharmaceutical achievements, strengthens the connection and trust between the consumers and pharmacists/pharmaceutical industry. 
Since personalization depends on the amount of personal information, in the proposed design it is limited to consumer's diagnosis, health condition and age. The consumers can retrieve the general information about their medication, its contraindications, precautions, and side effects. Also, they can gain personalized information regarding their health condition, tips about food supplements, diet, physical activities, and educational contents. All this information is tailored-based on the consumer diagnosis and age. For example, personalized information can help the consumer to be more aware regarding his/her health conditions, and consider food supplements (or diet) and activities that can help him/her to better manage and improve their health.

In order to have access to the features and personalized information of the MyMediMan (presented on Figure 2), the consumers need to register in the system. By creating their user profile, the consumers can save their latest search and read suggested contents. Also, the consumers can subscribe and receive the category of the information they choose (Health insights, Educational tips or News) by e-mail.

Several additional steps toward personalization of the proposed design will underpin and emphasize the consumer's role as a major actor in their own healthcare. The system should be deployed in several different languages. It will allow consumers to read the contents in their own language and thus better understand the information. An option for text-to-speech should also be implemented into the solution. As the number of users increases, more information can be gathered. This information can be used for better prediction and more accurate suggestions regarding the Health Insights and Educational Tips presented to the consumer. For this purpose, a recommendation algorithm for personalized suggestions about helpful multimedia contents can be incorporated.

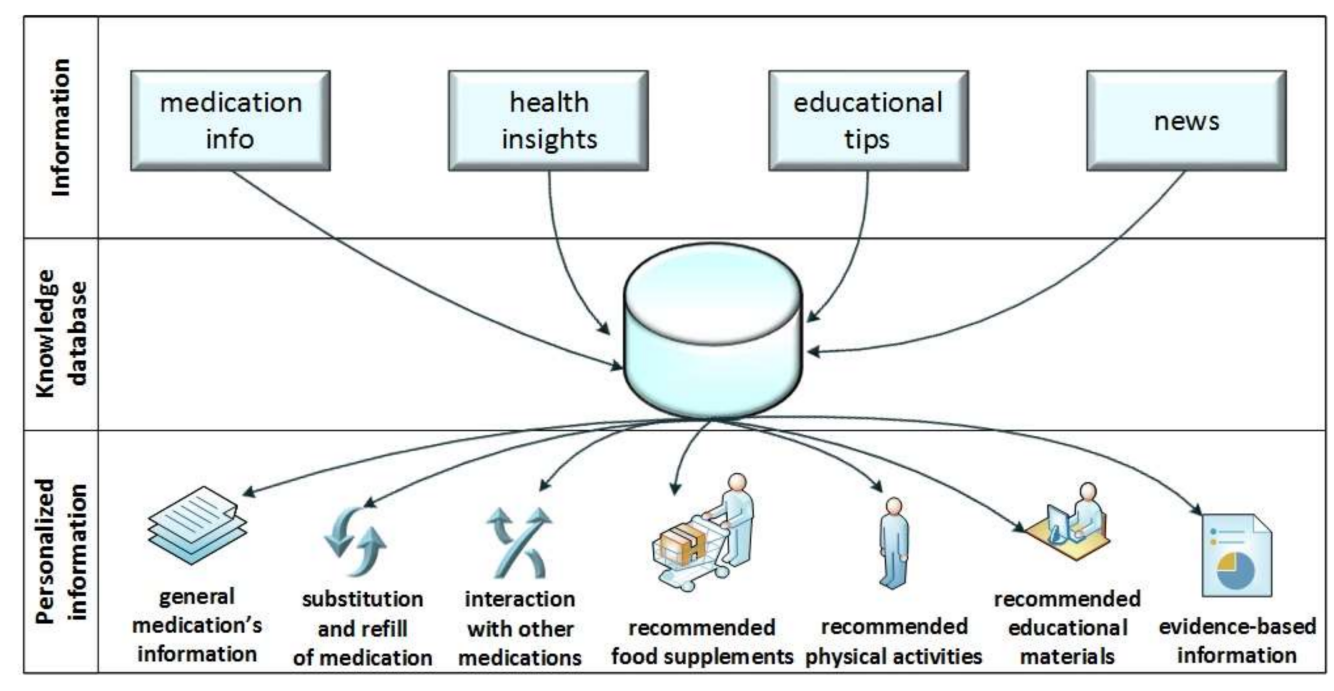

Figure 2. MyMediMan's personalized features and information.

In line with the concerns related to the privacy of the users and unauthorized advice, at this stage, no forum, chat or social media is incorporated into the design. In addition, the users are encouraged to seek professional advice for any concerns regarding their health condition.

\subsection{Use Case Scenarios}

The consumer can use the system in many ways. A general use case scenario is shown in the diagram on Figure 3.

In order to use the system, the consumer should register into it. During registration, the consumer inputs his/her name, surname, age, diagnosis (if applicable), e-mail, username and password. When the data is confirmed, the consumer is automatically logged into the system. If the consumer is already registered into the system, (s)he inputs his/her username and password. The system is running the process of authentication where the provided username and password are compared to 
those stored in the system. If the credentials match, the consumer is logged into the system. Otherwise, the system sends a message that the access is not granted and consumer needs to put the correct username and password. The processes of registration and authentication will use different algorithms and encryption techniques that will secure consumers' data privacy.

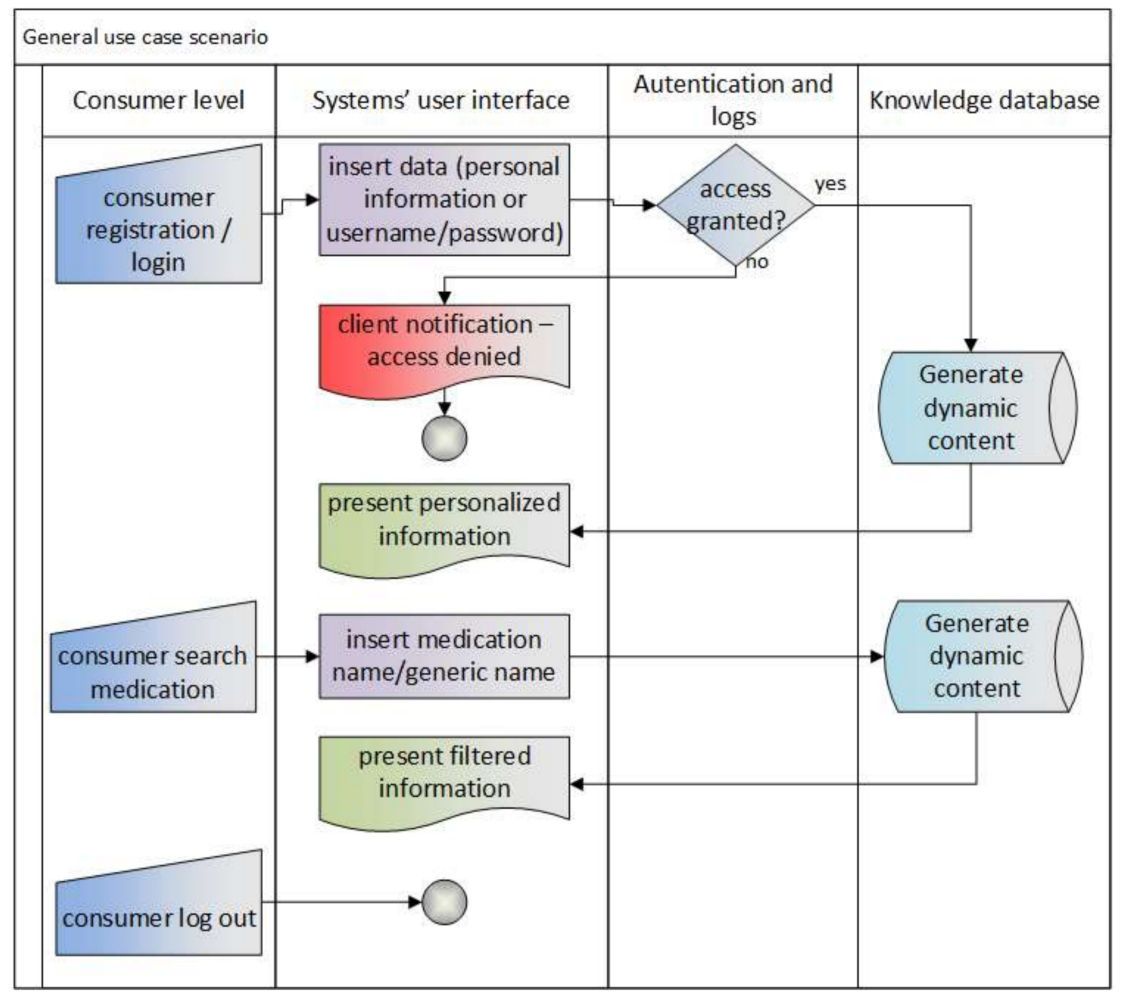

Figure 3. MyMediMan's general use case scenario.

The information presented to the consumer, after successful login to the system, is personalized based on the consumer's profile. The process of personalization will use filtering techniques and recommendation algorithms to customize presented information. All the health insights, educational tips and news presented on the home screen are personalized taking into account the consumer's age and diagnosis. The last 5 searches that the consumer has previously conducted are presented on the home screen.

With the search option, the consumer can look for information about particular medication using the name or generic name of that medication. Information regarding usage, dosage, contraindications, precautions, side effects, when not to use, substitution and refill of the medication are displayed in a new page (window). Additionally, below this information, news (provided by pharmaceutical industries) related to the searched medication is displayed.

At the end, the consumer should log out of the system.

\subsubsection{Use Case Scenario 1}

Mr. Brown registers into MyMediMan and inputs his/her name, surname, age, diagnosis, e-mail, username and password. He has diabetes. After successful registration, Mr. Brown is logged into the system and the user interface is updated. On the home screen are presented links to articles and other web pages that contain information about diets and physical activities that are appropriate for people with diabetes in his age group. In addition, there are tips for educational materials that provide convenient information about diabetes as a condition and how it can be managed. Several links related 
to the new trials and research from the pharmaceutical industries are also shown on the home screen. Since this is his first login, there is no information about his previous searches.

Educational materials for diabetes are very interesting and helpful for Mr. Brown. He wants to be informed about his condition and the ways he could manage it. He is making a subscription to receive the educational tips by e-mail. Mr. Brown logs out. Every week he receives an e-mail (from MyMediMan) with new links for educational materials related to diabetes.

\subsubsection{Use Case Scenario 2}

Mr. Brown is already registered in MyMediMan. He is using his username and password to log in. The home page displays health insights, educational tips and news based on his diagnosis and age group. Mr. Brown wants to read some information about the new medication the doctor prescribed him. He chooses the search option and inputs "metformin". In a new page, the system presents information about "metformin" (usage, dosage, contraindications, precautions, side effects, when it cannot be used, substitution and refill). After this information, links to articles regarding ongoing trials and research relevant to this medication are displayed. Mr. Brown does not have enough time to read all that information. He logs out.

After few days, Mr. Brown logins into MyMediMan. Besides all health insights, educational tips and news based on his diagnosis and age group, in the previous searches section is a link to his "metformin" search. Mr. Brown clicks on the link and new page with information about this medication is displayed. Mr. Brown continues with his reading.

\section{Conclusions and Future Work}

The main goal of the presented design is to introduce the consumers to different aspects of the medications they take and their overall health condition. By obtaining this information, the consumers will be aware of various medications' characteristics and different ways to improve their health, thus be more actively involved in their healthcare.

The information about medications should increase the consumers' consciousness towards the positive benefits of taking the medications. In addition, consumers should be aware of the consequences that particular medication can have on their health condition. Health insights and Educational Tips are offering various multimedia contents that are closely connected with different medications and conditions. This information allows the consumers to learn more about diverse medical conditions and ways of improving them. News from the pharmaceutical industry is a good starting point for widening consumers' perception of the process of medication development. This should strengthen the consumer confidence in medications and the entire pharmaceutical industry.

MyMediMan incorporates only core functionality of the medication management system, focusing on the personalization of the information presented to the consumer. The presented design can be broadened with additional modules that support medication adherence (like monitoring consumers' intake and generating reminders) and include medical personnel as an information provider and as a stakeholder of the system.

The presented study is only a research in progress in the field of personalization of medication management systems. The model is limited because it is based only on the opinion of the consumers and did not include any viewpoints of the other stakeholders. However, MyMediMan is a good base for implementation of the test (pilot) system that can be further expanded with additional features. An extensive evaluation that will include all stakeholders and assess different aspects of the presented system model MyMediMan is also one of the planned future developments of this study. Future research is needed for gathering evaluation results that will be analysed in order to determine the personal information that the users are willing to input in the medication management system, identify the personalized features needed, as well as personalized features acceptable by all stakeholders of the system. 
Author Contributions: All three authors collaboratively worked on this paper in different ways. Elena Vlahu-Gjorgievska, Khin T. Win and Willy Susilo conceived the idea; Elena Vlahu-Gjorgievska and Khin T. Win conducted the interviews and analysed the results; Willy Susilo and Elena Vlahu-Gjorgievska defined and analysed the model; Elena Vlahu-Gjorgievska wrote the paper. All authors have read and approved the final manuscript.

Conflicts of Interest: The authors declare no conflict of interest.

\section{References}

1. Win, K.T.; Hassan, N.M.; Bonney, A.; Iverson, D. Benefits of online health education: Perception from consumers and health professionals. J. Med. Syst. 2015, 39, 27. [CrossRef] [PubMed]

2. Cabana, M.D.; Le, T.T. Challenges in asthma patient education. J. Allergy Clin. Immunol. 2005, 115, $1225-1227$. [CrossRef] [PubMed]

3. Win, K.T.; Hassan, N.M.; Oinas-Kukkonen, H.; Probst, Y. Online patient education for chronic disease management: Consumer perspectives. J. Med. Syst. 2016, 40, 88. [CrossRef] [PubMed]

4. Kleppe, M.; Lacroix, J.; Ham, J.; Midden, C. A dual-process view on medication adherence: The role of affect. J. Health Psychol. 2017. [CrossRef] [PubMed]

5. IJsselsteijn, W.; de Kort, Y.; Midden, C.; Eggen, B.; van den Hoven, E. Persuasive Technology for Human Well-Being: Setting the Scene. In Proceedings of the 2006 1st International Conference on Persuasive Technology, Eindhoven, The Netherlands, 18-19 May 2006; Springer: Heidelberg/Berlin, Germany; Volume 3962, pp. 1-5.

6. IHamari, J.; Koivisto, J.; Pakkanen, T. Do Persuasive Technologies Persuade?-A Review of Empirical Studies. In Proceedings of the 9th International Conference on Persuasive Technology, Padua, Italy, 21-23 May 2014; Springer: Heidelberg/Berlin, Germany; Volume 8462, pp. 118-136.

7. Kaptein, M.; Markopoulos, P.; De Ruyter, B.; Aarts, E. Personalizing persuasive technologies: Explicit and implicit personalization using persuasion profiles. Int. J. Hum. Comput. Stud. 2015, 31, 38-51. [CrossRef]

8. Dijkstra, A. Personalization/Computer-Tailoring in Persuasive Technology: Tailoring Ingredients Target Psychological Processes. In Proceedings of the Personalization in Persuasive Technology Workshop, Persuasive Technology, Salzburg, Austria, 5 April 2016; pp. 6-12.

9. Busch, M.; Schrammel, J.; Tscheligi, M. Personalized Persuasive Technology—Development and Validation of Scales for Measuring Persuadability. In Proceedings of the 2013 8th International Conference on Persuasive Technology, Sydney, Austria, 3-5 April 2013; Springer: Berlin, Heidelberg; Volume 7822, pp. 33-38.

10. Blom, J. Personalization: A taxonomy. In Proceedings of the ACM CHI Conference on Human Factors in Computing Systems, Denver, CO, USA, 6-11 May 2017.

11. Oinas-Kukkonen, H.; Harjumaa, M. Persuasive systems design: Key issues, process model, and system features. Commun. Assoc. Inf. Syst. 2009, 24, 28.

12. Osborn, C.Y.; Rivet Amico, K.; Fisher, W.A.; Egede, L.E.; Fisher, J.D. An information-motivation-behavioral skills analysis of diet and exercise behavior in Puerto Ricans with diabetes. J. Health Psychol. 2010, 15, 1201-1213. [CrossRef] [PubMed]

13. Lee, M.K.; Kim, J.; Forlizzi, J.; Kiesler, S. Personalization revisited: A reflective approach helps people better personalize health services and motivates them to increase physical activity. In Proceedings of the 2015 ACM International Joint Conference on Pervasive and Ubiquitous Computing, Osaka, Japan, 7-11 September 2015; pp. 743-754.

14. Tara, M. Aspects of Information Tailoring in the 21st Century. In Encyclopedia of Information Science and Technology, 3rd ed.; IGI Global: New York, NY, USA, 2015; pp. 4042-4052.

15. Kreuter, M.W.; Strecher, V.J.; Glassman, B. One size does not fit all: The case for tailoring print materials. Ann. Behav. Med. 1999, 21, 276. [CrossRef] [PubMed]

16. Campbell, M.K.; Quintiliani, L.M. Tailored interventions in public health: Where does tailoring fit in interventions to reduce health disparities? Am. Behav. Sci. 2006, 49, 775-793. [CrossRef]

17. O’Connor, D.B.; Warttig, S.; Conner, M.; Lawton, R. Raising awareness of hypertension risk through a web-based framing intervention: Does consideration of future consequences make a difference? Psychol. Health Med. 2009, 14, 213-219. [CrossRef] [PubMed]

18. Ezendam, N.P.; Noordegraaf, V.S.; Kroeze, W.; Brug, J.; Oenema, A. Process evaluation of FATaintPHAT, a computer-tailored intervention to prevent excessive weight gain among Dutch adolescents. Health Promot. Int. 2012, 28, 26-35. [CrossRef] [PubMed] 
19. Kim, H.-Y.; Park, H.-A.; Min, Y.H.; Jeon, E. Development of an Obesity Management Ontology Based on the Nursing Process for the Mobile-Device Domain. J. Med. Int. Res. 2013, 15, e130. [CrossRef] [PubMed]

20. Granger, B.B.; Locke, S.C.; Bowers, M.; Sawyer, T.; Shang, H.; Abernethy, A.P.; Bloomfield, R.A., Jr.; Gilliss, C.L. The Digital Drag and Drop Pillbox: Design and Feasibility of a Skill-based Education Model to Improve Medication Management. J. Cardiovasc. Nurs. 2017, 32, E14. [CrossRef] [PubMed]

21. Teixeira, A.; Ferreira, F.; Almeida, N.; Silva, S.; Rosa, A.F.; Pereira, J.C.; Vieira, D. Design and development of Medication Assistant: Older adults centred design to go beyond simple medication reminders. Univ. Access Inf. Soc. 2017, 16, 545-560. [CrossRef]

22. Martin-Hammond, A.M.; Abegaz, T.; Gilbert, J.E. Designing an over-the-counter consumer decision-making tool for older adults. J. Biomed. Inf. 2015, 57, 113-123. [CrossRef] [PubMed]

23. Win, K.T.; Mullan, J.; Howard, S.; Oinas-Kukkonen, H. Persuasive systems design features in promoting medication management for consumers. In Proceedings of the 50th Hawaii International Conference on System Sciences, Koloa, HI, USA, 5-8 January 2016; pp. 3326-3335.

24. Ebner, H.; Modre-Osprian, R.; Kastner, P.; Schreier, G. Integrated Medication Management in mHealth Applications. Stud. Health Technol. Inf. 2014, 198, 238-244. [CrossRef]

25. Tschanz, M.; Dorner, T.L.; Denecke, K. eMedication Meets eHealth with the Electronic Medication Management Assistant (eMMA). Stud. Health Technol. Inf. 2017, 236, 196-203. [CrossRef]

(C) 2018 by the authors. Licensee MDPI, Basel, Switzerland. This article is an open access article distributed under the terms and conditions of the Creative Commons Attribution (CC BY) license (http://creativecommons.org/licenses/by/4.0/). 